\title{
Gauge-invariant coherent states for loop quantum gravity: I. Abelian gauge groups
}

\author{
Benjamin Bahr ${ }^{1}$ and Thomas Thiemann ${ }^{1,2}$ \\ ${ }^{1}$ MPI für Gravitationsphysik, Albert-Einstein Institut, Am Mühlenberg 1, 14467 Golm, Germany \\ 2 Perimeter Institute for Theoretical Physics, 31 Caroline St. N, Waterloo, Ontario N2L 2Y5, \\ Canada \\ E-mail: bbahr@aei.mpg.de and thomas.thiemann@aei.mpg.de
}

Received 13 October 2008, in final form 17 December 2008

Published 3 February 2009

Online at stacks.iop.org/CQG/26/045011

\begin{abstract}
In this paper, we investigate the properties of gauge-invariant coherent states for loop quantum gravity, for the gauge group $U(1)$. This is done by projecting the corresponding complexifier coherent states defined by Thiemann and Winkler to the gauge-invariant Hilbert space. This being the first step toward constructing physical coherent states, we arrive at a set of gauge-invariant states that approximate well the gauge-invariant degrees of freedom of Abelian loop quantum gravity (LQG). Furthermore, these states turn out to encode explicit information about the graph topology, and show the same pleasant peakedness properties known from the gauge-variant complexifier coherent states. In a companion paper, we will turn to the more sophisticated case of $S U(2)$.
\end{abstract}

PACS numbers: $02.10 . \mathrm{Ox}, 02.40 . \mathrm{Vh}, 04.60 . \mathrm{Pp}, 11.15 . \mathrm{Kc}$

\section{Introduction}

Loop quantum gravity (LQG) is a promising candidate for a theory that aims at combining the principles of quantum mechanics and general relativity (see [1-4] and references therein). The starting point of LQG is the Hamiltonian formulation of general relativity, choosing Ashtekar variables as phase-space coordinates, which casts GR into a $S U(2)$ gauge theory, leading to the Poisson structure

$$
\begin{aligned}
& \left\{A_{a}^{I}(x), A_{b}^{J}(y)\right\}=\left\{E_{I}^{a}(x), E_{J}^{b}(y)\right\}=0 \\
& \left\{A_{a}^{I}(x), E_{J}^{b}(y)\right\}=8 \pi G \beta \delta_{b}^{a} \delta_{J}^{I} \delta(x-y) .
\end{aligned}
$$

This system can be canonically quantized with the help of methods well known from algebraic quantum field theory, which results in a representation of the Poisson algebra on a Hilbert 
space $\mathcal{H}_{\text {kin }}$, which carries the kinematical information of quantum general relativity. In fact it has been shown that this is the only cyclic, irreducible representation if one demands the space diffeomorphisms to be unitarily implemented [5].

While the dynamics of classical general relativity is encoded into a set of phase-space functions $G_{I}, D_{a}, H$ that are constrained to vanish, these so-called constraints are, in LQG, promoted to operators that generate gauge transformations on the kinematical Hilbert space $\mathcal{H}_{\text {kin }}$. The physical Hilbert space $\mathcal{H}_{\text {phys }}$ is then to be derived as the set of vectors being invariant under these gauge transformations [6]:

$$
\hat{G}_{I}|\psi\rangle=\hat{D}_{a}|\psi\rangle=\hat{H}|\psi\rangle=0
$$

Although conceptually clear, the actual computation of $\mathcal{H}_{\text {phys }}$ is technically quite difficult. This is due to the fact that the constraints $\hat{G}_{I}, \hat{D}_{a}, \hat{H}$ act quite non-trivially on $\mathcal{H}_{\text {kin. }}$. Thus, while the kinematical setting is understood, the physical content of the theory is still unclear. It seems that, in its present formulation, LQG is too complicated to be solved analytically.

While this seems to be discouraging at first, complete solvability is not something one could have expected from the outset. In fact, nearly no theory which realistically describes a part of nature is completely solvable, neither in the quantum, nor in the classical regime. Rather, having the basic equations of a theory as a starting point, one has to develop tools for extracting knowledge about its properties in special cases, reducing the theory to simpler subsectors, approximate some solutions of the theory, or study its behavior via numerical methods. Examples for this range from reducing classical GR to symmetry-reduced situations, which is our main source of understanding the large-scale structure of our cosmos, over particle physics, where perturbational quantum field theory is our access to predict the behavior of elementary particles, to numerical simulations in ordinary quantum mechanics, which allow for computations of atomic and molecular spectra, transition amplitudes or band structures in solid state physics. Although in all of these fields the fundamental equations are well known, their solution is elusive, so one has to rely on approximations and numerics in order to understand the physical processes described by them. In other cases, such as interacting Wightman fields on 4D Minkowski space, not a single example is known to date. On the other hand, the perturbation theory for, say, $S U(N)$-Yang-Mills theory in small couplings is so effective that many particle physicists even regard the perturbative expansion in the coupling parameter as the fundamental theory in itself.

With these considerations, it seems quite natural to look for a way to gain knowledge about the physical content of LQG by approximative methods. One step into this direction has been done by introducing the complexifier coherent states.

For ordinary quantum mechanics, the well-known harmonic oscillator coherent states (HOCS)

$$
|z\rangle=\sum_{n=0}^{\infty} \frac{z^{n}}{\sqrt{n !}}|n\rangle
$$

are a major tool for performing analytical calculations and numerical computations. Not only can they be used to approximate quantum propagators [7], they are also the main tool for investigating the transition from quantum to classical behavior, as well as quantum chaos $[8,9]$. They also grant access to the numerical treatment of quantum dynamics for various systems $[10,11]$, and their generalization to quantum electrodynamics provides a path to the accurate description of laser light and quantum optics [12].

The complexifier coherent states (CCS), which have been first introduced in [13, 14], are a natural generalization of the HOCS to quantum mechanics on arbitrary compact Lie groups, 
and the complexifier methods employed to construct these states can also be transferred to other manifolds as well. Furthermore, for the special cases of quantum mechanics on the real line $\mathbb{R}$ and the circle $U(1)$, these states reduce to what has been used as coherent states for quite some time $[15,16]$.

In [17], the complexifier concept has been used to define complexifier coherent states for LQG. They are states on the kinematical Hilbert space $\mathcal{H}_{\text {kin }}$ and their properties have been exhibited in $[18,19]$. It was shown that they mimic the HOCS in their semiclassical behavior, in the sense that they describe the quantum system to be close to some point in the corresponding classical phase space of general relativity, minimizing relative fluctuations. Also, they provide a Bargman-Segal representation of $\mathcal{H}_{\text {kin }}$ as holomorphic functions, as well as approximating well quantum observables that correspond to classical phase-space variables.

This has indicated that these states are a useful tool for examining the semiclassical limit of LQG. In particular, it has been shown in [20] with the help of the CCS that the constraint operators for LQG, which are defined on $\mathcal{H}_{\text {kin }}$ and generate the dynamics of the theory, have the correct classical limit. In particular, CCS that are 'concentrated' around a classical solution of GR are annihilated by the constraint operators up to orders of $\hbar$. This indicates that, at least infinitesimally, LQG has classical GR as a semiclassical limit.

On the other hand, since the complexifier coherent states are only defined on $\mathcal{H}_{\text {kin }}$, none of them is really physical in the sense of the Dirac quantization programme. That is, while they are peaked on the classical constraint surface, they are not annihilated by the constraint operators, only approximately. Thus, while being a good tool for examining kinematical properties of LQG, it is not clear how well they approximate the dynamical aspects of quantum general relativity.

To do this, it would be desirable to have coherent states at hand that satisfy at least some of (1.2). We will pursue the first step on this path in this and the following paper.

Some of the constraints (1.2) are simpler than others. In particular, the easiest ones are the Gauss constraints $\hat{G}_{I}$. They are unbounded self-adjoint operators on $\mathcal{H}_{\text {kin }}$ and the gauge transformations generated by them are well understood. The set of vectors being invariant under the Gauss-gauge transformations ('gauge-invariant' in the following) is a proper subspace of $\mathcal{H}_{\text {kin }}$. This space is well known [21], and a basis for it is provided by the gauge-invariant spin-network functions, the construction of which involves intertwiners of the corresponding gauge group $S U(2)$. Thus, the straightforward way to construct gauge-invariant coherent states would be to project the CCS to the gauge-invariant Hilbert space.

The gauge transformations correspond to gauging the $S U(2)$-valued Ashtekar connection $A_{a}^{I}$ and its canonically conjugate, the electric flux $E_{I}^{a}$. Thus, the gauge group $S U(2)$ is involved, and in fact this group plays a prominent role in the construction of the whole kinematical Hilbert space $\mathcal{H}_{\text {kin. }}$. It is, however, possible to replace $S U$ (2) in this construction by any compact gauge group $G$, arriving at a different kinematical Hilbert space $\mathcal{H}_{\text {kin }}^{G}$, which would be the arena for the Hamiltonian formulation of a gauge field theory with gauge group $G$. Of course, one also has to replace the $\hat{G}_{I}$ by the corresponding gauge generators. The complexifier method is able to supply corresponding coherent states for each gauge group $G$.

This change of $S U(2)$ into another gauge group has been used frequently. In [22], it has been shown that the quantization of linearized gravity leads to the LQG framework with $U(1)^{3}$ as a gauge group. Furthermore, it has been pointed out [23] that changing $S U(2)$ for $U(1)^{3}$ does not change the qualitative behavior of the theory in the semiclassical limit, and so the $U(1)^{3}$-CCS have been used widely in order to investigate LQG [20].

Before treating the much more complicated case of $G=S U(2)$ in a sequel paper, in this paper we will, as a warm-up, consider the gauge group $G=U(1)$ and the corresponding CCS. The case $G=U(1)^{3}$ is then simply obtained by a triple tensor product: not only the 
kinematical Hilbert space

$$
\mathcal{H}_{\text {kin }}^{U(1)^{3}}=\mathcal{H}_{\text {kin }}^{U(1)} \otimes \mathcal{H}_{\text {kin }}^{U(1)} \otimes \mathcal{H}_{\text {kin }}^{U(1)}
$$

has this simple product structure, but also the respective gauge-invariant subspaces decompose according to (1.4). Also, $U(1)^{3}$-CCS are obtained by tensoring three $U(1)$-CCS. Due to this simple structure, it is sufficient for our arguments to consider the gauge-invariant coherent states in the case of $G=U(1)$, since all the properties revealed in this paper can be carried over straightforwardly to gauge-invariant coherent states for $G=U(1)^{3}$.

The plan for this paper is as follows: in chapter 2, we will shortly repeat the basics of LQG. In particular, the kinematical Hilbert space $\mathcal{H}_{\text {kin }}$ for arbitrary gauge group $G$ is defined, the corresponding set of constraints that generate the gauge transformations is described. In chapter 3, the complexifier coherent states are defined, where the focus lies on the particular case of $G=U(1)$. A formula for the inner product between two such states is derived, which depends purely on the geometry of the complexification of the gauge group $U(1)^{\mathbb{C}} \simeq \mathbb{C} \backslash\{0\}$.

In chapter 4 , we will apply the projector onto the gauge-invariant subspace of $\mathcal{H}_{\text {kin }}$ to the $U(1)$-complexifier coherent states. The involved gauge integrals can be carried out by a special procedure resembling gauge fixing. The resulting gauge-invariant states are then investigated, and their properties are displayed. In particular, we will show that they describe semiclassical states peaked at gauge-invariant degrees of freedom.

We will conclude this paper with a summary and an outlook on the following paper.

\section{The kinematical setting of LQG}

We shortly repeat the kinematical framework of LQG. Detailed expositions can be found in [1-4] and in the references therein.

The starting point of LQG is the phase space of the Ashtekar-connections $A_{a}^{I}(x)$ and the electric field $E_{J}^{b}(y)$, both defined on a 3-dim spatial manifold $\Sigma$, which can be thought of as a Cauchy surface in spacetime. The Poisson structure is given by (1.1). The fields $A_{a}^{I}(x), E_{J}^{b}(y)$ are not free, but subject to so-called constraints, which are phase-space functions. They encode the diffeomorphism invariance of the theory, and the Einstein equations. The reduced phase space consists of all phase space points $\left(A_{a}^{I}, E_{J}^{b}\right)$ where the constraints vanish. On this set, the constraints act as gauge transformations, and the set of gauge orbits is the physical phase space.

The set of constraints is divided into the Gauss constraints $G_{I}(x)$, the diffeomorphism constraints $D_{a}(x)$ and the Hamilton constraints $H(x)$. It is the set of Gauss constraints that is of particular importance in the rest of this work.

The holonomy-flux algebra generated by holonomies of $A_{a}^{I}(x)$ along edges and the electric fields $E_{J}^{b}(y)$ smeared over 2-dim surfaces is the starting point of the quantization programme. There is a unique cyclic representation of this algebra in which the spatial diffeomorphisms, which are generated by the diffeomorphism constraints $D_{a}(x)$, act unitarily and leave the vacuum state $\Omega$ invariant [5]. This kinematical Hilbert space $\mathcal{H}_{\text {kin }}$, on which the holonomyflux algebra is represented, also carries a representation of the constraint algebra, and is given by

$$
\mathcal{H}_{\text {kin }}=\bigoplus_{\gamma \in \Gamma} \mathcal{H}_{\gamma}
$$

Here, $\Gamma$ is the set of all graphs $\gamma$ in $\Sigma$ which consist of embedded, regular, analytic edges. Each Hilbert space $\mathcal{H}_{\gamma}$ is separable. If $\gamma$ is a graph with $E$ edges and $V$ vertices, $\mathcal{H}_{\gamma}$ is isomorphic to

$$
\mathcal{H}_{\gamma} \simeq L^{2}\left(G^{E}, \mathrm{~d} \mu_{H}^{\otimes E}\right)
$$


where $G$ is the gauge group acting on the fields $\left(A_{a}^{I}, E_{J}^{b}\right)$, and $d \mu_{H}$ is the normalized Haar measure on $G$. For LQG one uses $G=S U(2)$, or $G=U(1)^{3}$ for linearized LQG. In this paper, we will work with $G=U(1)$, before coming to $G=S U(2)$ in a companion paper.

Each of these $\mathcal{H}_{\gamma}$ is left invariant by the gauge transformations induced by the Gauss constraints $G_{I}(x)$. The restriction $\mathcal{G}_{\gamma}$ of the set of gauge transformations to $\mathcal{H}_{\gamma}$ is isomorphic to

$$
\mathcal{G}_{\gamma} \simeq G^{V}
$$

where $V$ is the number of vertices in the graph $\gamma$. The action of an element $\vec{k} \in G^{V}$ on a square-integrable function $\psi: G^{E} \rightarrow \mathbb{C}$ in $\mathcal{H}_{\gamma}$ is given by

$$
\alpha_{\vec{k}} \psi\left(h_{e_{1}}, \ldots, h_{e_{E}}\right):=\psi\left(k_{b\left(e_{1}\right)} h_{e_{1}} k_{f\left(e_{1}\right)}^{-1}, \ldots, k_{b\left(e_{E}\right)} h_{e_{E}} k_{f\left(e_{E}\right)}^{-1}\right),
$$

where $b\left(e_{m}\right)$ and $f\left(e_{m}\right)$ are the beginning and end points of the edge $e_{m}$. So, the gauge transformations act only at the vertices of a graph.

In particular, one can write down the projector onto the gauge-invariant Hilbert space for functions in $\mathcal{H}_{\gamma}$ :

$$
\begin{aligned}
\mathcal{P} \psi\left(h_{e_{1}}, \ldots, h_{e_{E}}\right) & :=\int_{G^{V}} \mathrm{~d} \mu_{H}\left(k_{1}, \ldots, k_{V}\right) \alpha_{k_{1}, \ldots k_{V}} \psi\left(h_{e_{1}} \ldots, h_{e_{E}}\right) \\
& =\int_{G^{V}} \mathrm{~d} \mu_{H}\left(k_{1}, \ldots, k_{V}\right) \psi\left(k_{b\left(e_{1}\right)} h_{e_{1}} k_{f\left(e_{1}\right)}^{-1}, \ldots, k_{b\left(e_{E}\right)} h_{e_{E}} k_{f\left(e_{E}\right)}^{-1}\right) .
\end{aligned}
$$

Since $G^{V}$ is compact, the integral exists and defines a projector

$$
\mathcal{P}: \mathcal{H}_{\gamma} \longrightarrow \mathcal{H}_{\gamma}
$$

onto a sub-Hilbert space of $\mathcal{H}_{\gamma}$. In particular, the gauge-invariant functions on a graph form a subset of all square-integrable functions on a graph. The gauge-invariant Hilbert spaces can be described using intertwiners between irreducible representations of $G$, and a basis for the gauge-invariant Hilbert spaces $\mathcal{P} \mathcal{H}_{\gamma}$ can be written down e.g. for $G=S U(2)$ in terms of gauge-invariant spin-network functions [21].

\section{Complexifier coherent states}

In the following, we will discuss, in brief, the complexifier method for obtaining coherent states for quantum mechanics on compact, semi-simple Lie groups $G$. We will then describe how to extend this procedure to obtain states for LQG.

\subsection{General gauge groups}

Consider quantum mechanics on a compact, semi-simple Lie group $G$, which is associated with the Hilbert space $L^{2}\left(G, \mathrm{~d} \mu_{H}\right)$, where $\mathrm{d} \mu_{H}$ is the normalized Haar measure on $G$. The classical configuration space is $G$, and the corresponding phase space is

$$
T^{*} G \simeq G^{\mathbb{C}} .
$$

Here, $G^{\mathbb{C}}$ is the complexification of $G$, generated by the complexification of the Lie algebra of $G, \mathfrak{g} \otimes \mathbb{C}$. Note that the diffeomorphism (3.1) is not unique, but can e.g. be determined by a non-negative phase-space function $C$, which grows more than linearly in the momenta. Such a function is called a complexifier, since it determines a complex structure on $T^{*} G$ via

$$
z(q, p)=\sum_{n=0}^{\infty} \frac{i^{n}}{n !} \underbrace{\{C, \ldots,\{C,\{C}_{n \text { times }}, p\} \ldots\},
$$


hence a diffeomorphism (3.1). The complexifier coherent states are then defined by

$$
\psi_{g}^{(\hat{C})}(h):=\left(\mathrm{e}^{-\hat{C}} \delta_{h^{\prime}}(h)\right)_{\left.\right|_{h^{\prime} \rightarrow g}} .
$$

The $\delta_{h^{\prime}}(h)$ is the delta distribution on $G$ with respect to $\mathrm{d} \mu_{H}$, centered around $h^{\prime} \in G, \hat{C}$ is the quantization of the complexifier function and $h^{\prime} \rightarrow g$ is the analytic continuation from $h^{\prime} \in G$ to $g \in G^{\mathbb{C}}$ as determined by the complex structure (3.2). The fact that the spectrum of $\hat{C}$ grows more than linearly in the momenta makes sure that the expression in the brackets is in fact a smooth function on $G$, thus ensuring that $\psi_{g}^{(\hat{C})} \in L^{2}\left(G, \mathrm{~d} \mu_{H}\right)$.

For the case of the Laplacian $\hat{C}=-\frac{t}{2} \Delta$ the complexifier coherent states can be expressed explicitly as

$$
\psi_{g}^{t}(h)=\sum_{\pi} \mathrm{e}^{-\lambda_{\pi} \frac{t}{2}} d_{\pi} \operatorname{tr} \pi\left(g h^{-1}\right),
$$

where the sum runs over all irreducible finite-dimensional representations $\pi$ of $G$. All characters $h \mapsto \operatorname{tr}(\pi(h))$ are eigenfunctions of the Laplacian $\Delta$ with eigenvalue $-\lambda_{\pi}<0$, and $d_{\pi}$ denotes the dimension of the representation $\pi$.

In the specific case of $G=U(1)$ and $G=S U(2)$, the states (3.4) have been investigated [17-19], and their properties are known quite well. In particular, they approximate the quantum analogs of holonomies and fluxes up to small fluctuations, the width of which is proportional to $\sqrt{t}$, which identifies $t$ as the parameter measuring the semiclassicality scale.

Since the kinematical Hilbert space $\mathcal{H}_{\text {kin }}$ of LQG consists of many copies of $\mathcal{H}_{\gamma} \simeq$ $\left(L^{2}\left(G, \mathrm{~d} \mu_{H}\right)\right)^{E}$, there are obvious candidates for coherent states in $\mathcal{H}_{\text {kin }}$, which are associated with a graph $\gamma=\left\{e_{1}, \ldots, e_{E}\right\}$. These are then given by a tensor product over all the edges $e_{k}$ of complexifier coherent states on $G$ :

$$
\psi_{g_{1}, \ldots, g_{E}}^{t}\left(h_{1}, \ldots, h_{E}\right)=\prod_{m=1}^{E} \psi_{g_{m}}^{t}\left(h_{m}\right) .
$$

The states (3.5) can also be obtained more systematically by choosing a complexifier for the phase space of GR. This choice also attaches a physical meaning of the parameters $g_{m} \in G^{\mathbb{C}}$ in terms of $A_{a}^{I}, E_{J}^{b}[17]$. In particular, $t$ measures the ratio

$$
t=\frac{\ell_{P}^{2}}{a^{2}}
$$

where $a$ is a characteristic length scale of the problem at hand. For kinematical states in LQG being close to some smooth spacetime, one expects $t$ to be of the order of $\ell_{P}^{2} /(1 \mathrm{~cm})^{2}$, i.e. about $10^{-70}$.

The choice of the semiclassicality parameter $t$ being equal for all edges $e_{m}$ of the graph $\gamma$ is by no means a necessity. In particular, a varying choice is probably more realistic, e.g. if one wants to describe spaces in which a small region exhibits large quantum fluctuations in the metric, while the rest is close to classical, smooth GR. For simplicity, we choose all $t_{m}$ equal: $t_{m} \equiv t$. All qualitative arguments in this paper carry over to the more general case of edge-dependent $t$.

The complexified groups $G^{\mathbb{C}}$ are diffeomorphic to the tangent bundle of the groups $T^{*} G$ themselves. So, the complexifier coherent states are labeled by elements of the classical phase space. A state labeled by $g_{1}, \ldots, g_{E}$ corresponds to a state being close to the classical phase-space point corresponding to $g_{1}, \ldots, g_{E}$. This interpretation is supported by the fact that - as could be shown for the cases $G=U(1)$ and $G=S U(2)$ - the expectation values of quantizations of holonomies and fluxes coincide - up to corrections in $t$-with the classical 
holonomies and fluxes determined by the phase-space point corresponding to $g_{1}, \ldots, g_{E}[19]$. Furthermore, the overlap between two complexifier coherent states is sharply peaked

$$
\frac{\|\left.\left\langle\psi_{g_{1}, \ldots, g_{E}}^{t} \mid \psi_{g_{1}^{\prime}, \ldots, g_{E}^{\prime}}^{t}\right\rangle\right|^{2}}{\left\|\psi_{g_{1}, \ldots, g_{E}}^{t}\right\|^{2}\left\|\psi_{g_{1}^{\prime}, \ldots, g_{E}^{\prime}}^{t}\right\|^{2}}=\left\{\begin{array}{cc}
1 & g_{m}=g_{m}^{\prime} \text { for all } m \\
\text { decaying exponentially } & \text { else. }
\end{array}\right.
$$

This shows that the complexifier coherent states (3.4) are suitable to approximate the kinematical operators of LQG quite well.

\subsection{The case of $G=U(1)$}

In the last section, the general definition of complexifier coherent states for arbitrary compact Lie groups $G$ has been given. In this section, we will shortly review these states for the simplest case of $G=U(1)$, since we will work with these states in the rest of this paper.

From (3.4), we can immediately deduce the explicit form of the complexifier coherent states, since all irreducible representations of $U(1)$ are known and one-dimensional

$$
\psi_{z}^{t}(\phi)=\sum_{n \in \mathbb{Z}} \mathrm{e}^{-n^{2} \frac{t}{2}} \mathrm{e}^{-\mathrm{i} n(z-\phi)}
$$

for $g=\mathrm{e}^{\mathrm{i} z}$ and $h=\mathrm{e}^{\mathrm{i} \phi}$. With the Poisson summation formula, this expression can be rewritten as

$$
\psi_{z}^{t}(\phi)=\sqrt{\frac{2 \pi}{t}} \sum_{n \in \mathbb{Z}} \mathrm{e}^{-\frac{(z-\phi-2 \pi n)^{2}}{2 t}} .
$$

The inner product of the two of these states is then

$$
\left\langle\psi_{w}^{t} \mid \psi_{z}^{t}\right\rangle=\sqrt{\frac{\pi}{t}} \sum_{n \in \mathbb{Z}} \mathrm{e}^{-\frac{(\bar{w}-z-2 \pi n)^{2}}{4 t}}=\psi_{\bar{w}-z}^{2 t}(0) .
$$

There is a way to interpret (3.9) geometrically. This makes use of the fact that $G^{\mathbb{C}}=\mathbb{C} \backslash\{0\}$ comes with a pseudo-Riemannian metric provided by the Killing form on its Lie algebra. On arbitrary Lie groups $G$, this metric is denoted, in components, by

$$
h_{I J}=-\frac{1}{\operatorname{dim} G} \operatorname{tr}\left(g^{-1} \partial_{I} g g^{-1} \partial_{J} g\right) .
$$

Choosing the chart $z \rightarrow \mathrm{e}^{\mathrm{i} z}$ on $\mathbb{C} \backslash\{0\}$, the metric (3.10) simply takes the form $h=1$. Note that the geodesics through $1 \in \mathbb{C} \backslash\{0\}$ with respect to this metric are given by

$$
t \longmapsto \mathrm{e}^{\mathrm{i} t z}
$$

for some $z \in \mathbb{C}$, which corresponds to the velocity of the geodesic at $t=0$. Note also that geodesics can be transported via group multiplication, since the metric is defined via group translation. In particular, if $\gamma(t)$ is a geodesic on $\mathbb{C} \backslash\{0\}$, then $g \gamma(t)$ is also one for any $g \in \mathbb{C} \backslash\{0\}$.

With $h$ one can define the complex length square of a geodesic, or any other regular curve $\gamma$ on $\mathbb{C} \backslash\{0\}$, via

$$
l^{2}(\gamma):=\left(\int \mathrm{d} t \sqrt{h(\gamma(t)) \dot{\gamma}(t) \dot{\gamma}(t)}\right)^{2} .
$$

Note that this gives a well-defined complex number, since the square root of a complex number is defined up to a sign, and this sign is chosen continuously on the whole curve, which gives a unique choice since the curve is regular, i.e. its velocity vector vanishes nowhere. So, the integral is determined up to a sign, the square of which is then well defined. 
Let $g, h \in \mathbb{C} \backslash\{0\}$, and $\gamma:[0,1] \rightarrow \mathbb{C} \backslash\{0\}$ be a geodesic from $g$ to $h$. It is straightforward to compute that such a geodesic is not unique, but, for $g=\mathrm{e}^{\mathrm{i} w}$ and $h=\mathrm{e}^{\mathrm{i} z}$ (where $z$ and $w$ are determined up to $2 \pi n$ for some $n \in \mathbb{Z}$ ), is given by

$$
\gamma(t)=\mathrm{e}^{\mathrm{i} w} \mathrm{e}^{\mathrm{i} t(z-w-2 \pi n)},
$$

for any $n \in \mathbb{Z}$. By changing $n$, one ranges through the set of geodesics from $g$ to $h$. The complex length square of the (3.13) can easily be computed to be

$$
l(\gamma)^{2}=(z-w-2 \pi n)^{2} .
$$

This shows that one can write the inner product between two complexifier coherent states as sum over complex lengths of geodesics

$$
\left\langle\psi_{g}^{t} \mid \psi_{h}^{t}\right\rangle=\sqrt{\frac{\pi}{t}} \sum_{\substack{\gamma \text { geodesic } \\ \text { from } g^{c} \text { to } h}} \mathrm{e}^{-\frac{l(\gamma)^{2}}{4 t}},
$$

with $g^{c}:=\bar{g}^{-1}$.

Here one can see that the infinite sum in (3.9) has the interpretation of a sum over geodesics connecting two elements on phase space $U(1)^{\mathbb{C}} \simeq \mathbb{C} \backslash\{0\}$. As will be shown in the following paper, the inner product of two complexifier coherent states for $G=S U(2)$ can be written in an analogous way, which suggests that this is a common feature independent of the gauge group.

\section{Gauge-invariant coherent states with gauge group $G=U(1)$}

\subsection{The gauge-invariant sector}

In the following, we will describe the Hilbert space invariant under the Gauss gauge transformation group. Since this gauge transformation group $\mathcal{G}$ leaves every graph invariant, we can restrict ourselves to the case of one graph, in particular

$$
\mathcal{P} \underset{\lim }{\longrightarrow} H_{\gamma}=\underset{\lim }{\longrightarrow} \mathcal{P} \mathcal{H}_{\gamma}
$$

So, we can consider the gauge-invariant cylindrical functions on each graph separately.

The gauge-invariant cylindrical functions on a graph $\gamma$ with $E$ edges and $V$ vertices can be described in terms of singular cohomology classes with values in the gauge group. In particular, every Hilbert space $\mathcal{H}_{\gamma}$ is canonically isomorphic to an $L^{2}$-space

$$
H_{\gamma} \simeq L^{2}\left(G^{E}, \mathrm{~d} \mu_{H}^{\otimes E}\right),
$$

where $d \mu_{H}$ is the normalized Haar measure on the compact Lie group $G$. It is known that the gauge-invariant Hilbert space is then canonically isomorphic to an $L^{2}$-space over the first simplicial cohomology group of $\gamma$ with values in the gauge group $G$

$$
\mathcal{P} H_{\gamma} \simeq L^{2}\left(H^{1}(\gamma, G), \mathrm{d} \mu\right)
$$

with a certain measure $\mathrm{d} \mu$. For Abelian gauge groups $G$, the first cohomology group of $\gamma$ with values in $G$ is given by

$$
H^{1}(\gamma, G) \simeq G^{E-V+1}
$$

and $\mathrm{d} \mu=\mathrm{d} \mu_{H}^{\otimes E-V+1}$ is the $E-V+1$-fold tensor product of the Haar measure on $G$. For non-Abelian gauge groups $G$ a similar result holds, while the definition of the first cohomology class requires more care. This case will be dealt with in the following paper, and we stay with Abelian $G$ in this paper. 


\subsection{Gauge-invariant coherent states}

We now come to the main part of this paper: the computation of the gauge-invariant coherent states. We will derive a closed form for them, revealing the intimate relationship between the gauge-invariant degrees of freedom and the graph topology. From the explicit form we will be able to compute the overlap between two gauge-invariant coherent states, which will allow for an interpretation as semiclassical states for the gauge-invariant sector of the theory.

The gauge-invariant coherent states are obtained by applying the gauge projector (2.5) to the complexifier coherent states on a graph (3.5), (3.7), i.e.

$$
\Psi_{\left[g_{1}, \ldots, g_{E}\right]}^{t}\left(\left[h_{1}, \ldots h_{E}\right]\right)=\mathcal{P} \psi_{g_{1}, \ldots, g_{E}}^{t}\left(h_{1}, \ldots, h_{E}\right) .
$$

It is known that the set of gauge-invariant functions can be described in terms of functions on the first cohomology class of the graph. In particular, if the graph has $E$ edges and $V$ vertices, i.e. the gauge-variant configuration space is diffeomorphic to $U(1)^{E}$, then the gauge-invariant configuration space is diffeomorphic to $U(1)^{E-V+1}$. This might raise the hope that these states somehow resemble complexifier coherent states on the gauge-invariant configuration space $U(1)^{E-V+1}$. We will see that this is not quite true, but near enough.

The fact that the gauge group is Abelian is a great simplification: it allows us to pull back all group multiplications to simple addition on the algebra, simply due to the fact that $\operatorname{expiz} \operatorname{expi} w=\operatorname{expi}(z+w)$. This will allow us to explicitly perform the gauge integrals for arbitrary graphs, and obtain a formula for the gauge-invariant coherent states that only depends on gauge-invariant combinations of $h_{k}=\operatorname{expi} \phi_{k}$ and $g_{k}=\operatorname{expi} z_{k}$, as well as topological information about the graph, in particular its incidence matrix.

\subsection{Basic graph theory}

In order to be able to deal with the expressions for all graphs, we start with some basics of graph theory. All the material, as well as all the proofs, can be found in [24] and the references therein.

Definition 4.1. Let $\gamma$ be a directed graph with $V$ vertices and $E$ edges. Let the edges be labeled by numbers $1, \ldots, E$ and the vertices by numbers $1, \ldots, V$. Then the incidence matrix $\lambda \in \operatorname{Mat}(E \times V, \mathbb{Z})$ is defined by the following rule:

$$
\begin{array}{ll}
\lambda_{k l}:=1 & \text { if the edge } k \text { ends at vertex } l \\
\lambda_{k l}:=-1 & \text { if the edge } k \text { starts at vertex } l \\
\lambda_{k l}:=0 & \text { else. }
\end{array}
$$

Note in particular that, if edge $k$ starts and ends at vertex $l$, i.e. the edge $k$ is a loop, then $\lambda_{k l}=0$ as well. Since either an edge is a loop or starts at one and ends at some other vertex, every line of the matrix $\lambda$ is either empty, or contains exactly one 1 and one -1 . With the definition

$$
u:=\left(\begin{array}{c}
1 \\
1 \\
\vdots \\
1
\end{array}\right) \in \mathbb{R}^{V}
$$

we immediately conclude

$$
\lambda^{T} u=0 \text {. }
$$


Definition 4.2. Let $\gamma^{\prime}$ be a graph. If $\gamma^{\prime}$ contains no loops, then $\gamma^{\prime}$ is said to be a tree. If $\gamma^{\prime} \subset \gamma$ is a subgraph, then $\gamma^{\prime}$ is said to be a tree in $\gamma$. If $\gamma^{\prime} \subset \gamma$ is a subgraph that meets every vertex of $\gamma$, then $\gamma^{\prime}$ is said to be a maximal tree (in $\gamma$ ).

Lemma 4.1. Every graph $\gamma$ has a maximal tree as subgraph. Every tree has $V=E+1$ vertices.

Maximal trees in graphs are not unique. It is quite easy to show that every function cylindrical over a graph $\gamma$ is gauge equivalent to a function cylindrical over $\gamma$, which is constant on the edges corresponding to a maximal tree. This will be used later, and by the preceding lemma we immediately conclude that the gauge-invariant degree of freedoms on a graph with $V$ vertices and $E$ edges is $E-V+1$ for Abelian gauge theories. This will be seen explicitly at the end of this section.

The following theorem relates the numbers of different possible maximal trees to the incidence matrix:

Theorem 4.1 (Kirchhoff). Let $\gamma$ be a graph and $\lambda$ its incidence matrix. Then the Kirchoffmatrix $K:=\lambda \lambda^{T}$ has nonnegative eigenvalues

$$
0=\mu_{1} \leqslant \mu_{2} \leqslant \cdots \leqslant \mu_{V} .
$$

The lowest eigenvalue is $\mu_{1}=0$, and the degeneracy of 0 is the number of connected components of the graph $\gamma$. Furthermore, the product of all nonzero eigenvalues

$$
G:=\frac{1}{V} \prod_{\mu_{k} \neq 0} \mu_{k}
$$

is the number of different maximal trees in $\gamma$.

With this machinery, we will be able to perform the gauge integral for arbitrary graphs. This will include some kind of gauge-fixing procedure, which will make use of a maximal tree.

\subsection{Gauge-variant coherent states and the gauge integral}

The Abelianness of the gauge group allows us to pull back the group multiplication to addition on the Lie algebra. This is why throughout this chapter we will, instead of elements $h \in U(1)$, deal with $\phi \in \mathbb{R}$ by $h=\exp i \phi$, and instead of elements $g \in \mathbb{C} \backslash\{0\}$, we will work with the corresponding $z \in \mathbb{C}$ such that $g=\exp i z$, always having in mind that $\phi$ and $z$ are only defined modulo $2 \pi n$ for $n \in \mathbb{Z}$.

We will denote vectors (of any length) as simple letters $z, \phi, \tilde{\phi}, m, \ldots$ and their various components with indices: $z_{k}, \phi_{k}, \tilde{\phi}_{k}, \ldots$. The particular range of the indices will be clear from the context, but we will still repeat it occasionally.

The gauge-variant coherent states on a graph $\gamma$ with $E$ edges are simply given by the product

$$
\psi_{z}^{t}(\phi)=\prod_{k=1}^{E} \sum_{m_{k} \in \mathbb{Z}} \mathrm{e}^{-m_{k}^{2} \frac{t}{2}} \mathrm{e}^{\mathrm{i} m_{k}\left(z_{k}-\phi_{k}\right)},
$$

where $z_{k}=\varphi_{k}-\mathrm{i} p_{k}, k=1, \ldots, E$ is labeling the points in phase space where the coherent states are peaked. With the Poisson summation formula one can rewrite this as

$$
\psi_{z}^{t}(\phi)=\sqrt{\frac{2 \pi}{t}}^{E} \sum_{m_{1}, \ldots, m_{E} \in \mathbb{Z}} \exp \left(-\sum_{k=1}^{E} \frac{\left(z_{k}-\phi_{k}-2 \pi m_{k}\right)^{2}}{2 t}\right) .
$$


We will now perform the gauge integral

$$
\begin{aligned}
\Psi_{[z]}^{t}(\phi) & =\int_{U(1)^{V}} \mathrm{~d} \mu_{H}(\tilde{\phi}) \psi_{\alpha_{\tilde{\phi} z}}^{t}(\phi) \\
& =\sqrt{\frac{2 \pi}{t}} \int_{[0,2 \pi]^{V}} \frac{\mathrm{d} \tilde{\phi}_{1}}{2 \pi} \cdot \ldots \cdot \frac{\mathrm{d} \tilde{\phi}_{V}}{2 \pi} \sum_{m_{1}, \ldots, m_{E} \in \mathbb{Z}} \exp \left(-\sum_{k=1}^{E} \frac{\left(A_{k}+\lambda_{k a} \tilde{\phi}_{a}-2 \pi m_{k}\right)^{2}}{2 t}\right)
\end{aligned}
$$

with $A=z-\phi$, and where $\lambda_{k a}$ are the components of the transpose $\lambda^{T}$ of the incidence matrix.

In what follows, we will use the symmetries of this expression, together with a gaugefixing procedure, to separate the gauge degrees of freedom from the gauge-invariant ones. The integrals will then be performable analytically, and the resulting expression can then be interpreted as states being peaked on gauge-invariant quantities.

To simplify the notation, we will assume, without loss of generality, that $\gamma$ is connected. Furthermore choose, once and for all, a maximal tree $\tau \subset \gamma$. Choose the numeration of vertices and edges of $\gamma$ according to the following scheme:

Start with the maximal tree $\tau$. The tree consists of $V$ vertices and $V-1$ edges. Call a vertex that has only one outgoing edge (in $\tau$, not necessarily in $\gamma$ ) an outer end of $\tau$. Remove one outer end and the corresponding edge from $\tau$ and obtain a smaller subgraph $\tau^{\prime} \subset \gamma$, which is also a tree. Label the removed vertex with the number 1, and do so with the removed edge as well. So this gives you $v_{1}$ and $e_{1}$. From $\tau^{\prime}$, remove an outer end and the corresponding edge, and label them $v_{2}$ and $e_{2}$, and obtain a yet smaller tree $\tau^{\prime \prime} \subset \tau^{\prime} \subset \tau \subset \gamma$. Repeat this process until $\tau$ has been reduced to $\tau^{(V-1 \text { dashes })}$, which is a point. This way, one has obtained $v_{1}, \ldots, v_{V-1}$ and $e_{1}, \ldots, e_{V-1}$. Call the last remaining vertex $v_{V}$. Label the edges that do not belong to $\tau$ by $e_{V}, e_{V+1}, \ldots, e_{E}$ in any order.

Choosing the numeration of the vertices and the edges in the above manner will help us in rewriting the expression (4.9). First we note that the first $V-1$ edges and the first $V$ vertices constitute the tree, the last $E-V+1$ edges constitute what is not the tree in $\gamma$. Furthermore, with this numeration, the edge $e_{k}$ is starting or ending at vertex $v_{k}$ for $k=1, \ldots, V-1$. In particular, the diagonal elements of the incidence matrix are all nonzero: $\lambda_{k k} \neq 0$ for $k=1, \ldots, V-1$.

Definition 4.3. Let $\gamma$ be a graph, with vertices $v_{1}, \ldots v_{V}$ and edges $e_{1}, \ldots, e_{E}$. Between two vertices $v_{k}$ and $v_{l}$ there is a unique path in $\tau$, since a tree contains no loops. Call $v_{k}$ being before $v_{l}$, if this path includes $e_{k}$, otherwise call $v_{k}$ being after $v_{l}$.

Note that a vertex cannot be both before and after another vertex, but two vertices can both be before or both be after each other.

The numeration we have chosen has the following consequence: for each vertex $v_{k}$ one has that for all $v_{l}$ such that $v_{k}$ is after $v_{l}$, that $l \leqslant k$. The converse need not be true. Note further that every vertex is before itself, by this definition. Also, since $e_{V}$ is not an edge of the graph, it does not even have to be touching $v_{V}$. So, the question of whether $v_{V}$ is before or after any other vertex makes no sense in this definition (but note that it does make sense to ask whether any vertex is before or after $v_{V}$ ).

We now rewrite formula (4.9), by replacing the integrals over $[0,2 \pi]$ by integrals over $\mathbb{R}$. We do this inductively over the vertices from $v_{1}$ to $v_{V-1}$.

Consider the $E$ terms constituting the sum in the exponent in (4.9). In some of them $\tilde{\phi}_{1}$ appears, in some of them it does not, precisely if either $\lambda_{k 1} \neq 0$ or $\lambda_{k 1}=0$. Note that $\tilde{\phi}_{1}$ definitely appears in the first term, by the above considerations. If $\tilde{\phi}_{1}$ appears in the $k$ th 
term other that $k=1$, shift the infinite sum over $m_{k}$ by $m_{k}+\lambda_{11} \lambda_{k 1} m_{1}$. The result of this is that, since $\lambda_{k 1}^{2}=\lambda_{11}^{2}=1$ for these $k$, after this shift $\tilde{\phi}_{1}$ appears always in the combination $\lambda_{11} \tilde{\phi}_{1}-2 \pi m_{1}$ in all the factors. Now we can employ the formula

$$
\int_{[0,2 \pi]} \frac{\mathrm{d} \tilde{\phi}}{2 \pi} \sum_{m \in \mathbb{Z}} f(\tilde{\phi} \pm 2 \pi m)=\frac{1}{2 \pi} \int_{\mathbb{R}} \mathrm{d} \tilde{\phi} f(\tilde{\phi})
$$

and, regardless of whether $\lambda_{11}=+1$ or $\lambda_{l l}=-1$, have

$$
\begin{aligned}
\text { (4.9) }=\sqrt{\frac{2 \pi}{t}}^{E} & \int_{\mathbb{R}} \frac{\mathrm{d} \tilde{\phi}_{1}}{2 \pi} \int_{[0,2 \pi]^{V-1}} \frac{\mathrm{d} \tilde{\phi}_{2}}{2 \pi} \cdot \ldots \cdot \frac{\mathrm{d} \tilde{\phi}_{V}}{2 \pi} \\
& \times \sum_{m_{2}, \ldots, m_{E} \in \mathbb{Z}} \exp \left(-\frac{\left(A_{1}+\lambda_{1 a} \tilde{\phi}_{a}\right)^{2}}{2 t}-\sum_{k=2}^{E} \frac{\left(A_{k}+\lambda_{k a} \tilde{\phi}_{a}-2 \pi m_{k}\right)^{2}}{2 t}\right) .
\end{aligned}
$$

This being the beginning of the induction, we now describe the induction step from $l$ to $l+1$ by the following technical lemma. By this we will be able to extend all integration ranges over all of $\mathbb{R}$, instead of finite intervals, which will turn out to be very useful.

Lemma 4.2. Let $\gamma$ be a graph with $V$ vertices, $E$ edges and $\lambda$ be its incidence matrix. Let $A \in \mathbb{C}^{E}$ and $t>0$, then we have, for $1 \leqslant l \leqslant V-1$ :

$$
\begin{aligned}
\sqrt{\frac{2 \pi}{t}} \int_{\mathbb{R}^{l-1}} \frac{\mathrm{d} \tilde{\phi}_{1}}{2 \pi} & \ldots \frac{\mathrm{d} \tilde{\phi}_{l-1}}{2 \pi} \int_{[0,2 \pi]^{V-l+1}} \frac{\mathrm{d} \tilde{\phi}_{l}}{2 \pi} \cdot \ldots \cdot \frac{\mathrm{d} \tilde{\phi}_{V}}{2 \pi} \\
& \times \sum_{m_{l}, \ldots, m_{E} \in \mathbb{Z}} \exp \left(-\sum_{k=1}^{l-1} \frac{\left(A_{k}+\lambda_{k a} \tilde{\phi}_{a}\right)^{2}}{2 t}-\sum_{k=l}^{E} \frac{\left(A_{k}+\lambda_{k a} \tilde{\phi}_{a}-2 \pi m_{k}\right)^{2}}{2 t}\right) \\
= & \sqrt{\frac{2 \pi}{t}} \int_{\mathbb{R}^{l}} \frac{\mathrm{d} \tilde{\phi}_{1}}{2 \pi} \cdot \ldots \cdot \frac{\mathrm{d} \tilde{\phi}_{l}}{2 \pi} \int_{[0,2 \pi]^{V-l}} \frac{\mathrm{d} \tilde{\phi}_{l+1}}{2 \pi} \cdot \ldots \cdot \frac{\mathrm{d} \tilde{\phi}_{V}}{2 \pi} \\
& \times \sum_{m_{l+1}, \ldots, m_{E} \in \mathbb{Z}} \exp \left(-\sum_{k=1}^{l} \frac{\left(A_{k}+\lambda_{k a} \tilde{\phi}_{a}\right)^{2}}{2 t}-\sum_{k=l+1}^{E} \frac{\left(A_{k}+\lambda_{k a} \tilde{\phi}_{a}-2 \pi m_{k}\right)^{2}}{2 t}\right) .
\end{aligned}
$$

Proof. Note that we just proved the formula for $l=1$. In the proof for arbitrary $1 \leqslant l \leqslant V-1$ we will use the notion of vertices being before and after one another.

Consider all vertices $v_{k}$ such that $v_{l}$ is after $v_{k}$. By construction, for all such $k$, we have $k<l$, so by the induction hypothesis, the integration over all these $v_{k}$ runs over all of $\mathbb{R}$, not over just the interval $[0,2 \pi]$ any more. Consequently, the sum over these $m_{k}$ is not appearing any longer. So we can shift the integration range by $+2 \pi \lambda_{l l} m_{l}$.

This will affect the terms in the first sum in

$$
\exp \left(-\sum_{k=1}^{l-1} \frac{\left(A_{k}+\lambda_{k a} \tilde{\phi}_{a}\right)^{2}}{2 t}-\sum_{k=l}^{E} \frac{\left(A_{k}+\lambda_{k a} \tilde{\phi}_{a}-2 \pi m_{k}\right)^{2}}{2 t}\right)
$$

the following way: Let $k<l$. The edge $e_{k}$ then belongs to the tree $\tau$, and thus $v_{l}$ is either after both vertices $e_{k}$ touches, or before both vertices. If $v_{l}$ is before both, the term does not change at all, since the two $\tilde{\phi}_{a}$ in it are not shifted. If $v_{l}$ is after both and is not itself one of the two vertices, then the term gets changed by

$$
\left(A_{k}+\lambda_{k a} \tilde{\phi}_{a}\right)^{2} \longrightarrow\left(A_{k}+\lambda_{k a} \tilde{\phi}_{a} \pm 2 \pi \lambda_{l l} m_{l} \mp 2 \pi \lambda_{l l} m_{l}\right)^{2}=\left(A_{k}+\lambda_{k a} \tilde{\phi}_{a}\right)^{2},
$$


since the two $\tilde{\phi}_{a}$ in a term always appear with opposite sign. So these terms do not change, too. If $v_{l}$ is after both vertices that touch $e_{k}$ and is itself one of it (i.e. $e_{k}$ is an edge adjacent to $e_{l}$, linked by $v_{l}$ ), then the corresponding term changes by

$$
\begin{aligned}
\left(A_{k}+\lambda_{k a} \tilde{\phi}_{a}\right)^{2} & =\left(A_{k}+\lambda_{k l} \tilde{\phi}_{l}+\lambda_{k k} \tilde{\phi}_{k}\right)^{2}=\left(A_{k}+\lambda_{k k}\left(\tilde{\phi}_{k}-\tilde{\phi}_{l}\right)\right)^{2} \\
& \longrightarrow\left(A_{k}+\lambda_{k k}\left(\tilde{\phi}_{k}-\tilde{\phi}_{l}+2 \pi \lambda_{l l} m_{l}\right)\right)^{2},
\end{aligned}
$$

where $\lambda_{k l}=-\lambda_{l l}$ and $\lambda_{l l}^{2}=1$ have been used.

So, after this shift, in all terms in the first sum in (4.12) $\tilde{\phi}_{l}$ has been replaced by $\tilde{\phi}_{l}-2 \pi \lambda_{l l} m_{l}$. The first term of the second sum reads

$$
\left(A_{l}+\lambda_{l l}\left(\tilde{\phi}_{l}-\tilde{\phi}_{a}\right)-2 \pi m_{l}\right)^{2}=\left(A_{l}-\lambda_{l l} \tilde{\phi}_{a}+\lambda_{l l}\left(\tilde{\phi}_{l}-2 \pi \lambda_{l l} m_{l}\right)\right)^{2},
$$

where $v_{a}$ is the other vertex touching $e_{l}$, apart from $v_{l}$. So also in this term $\tilde{\phi}_{l}$ and $m_{l}$ appear in the combination $\tilde{\phi}_{l}-2 \pi \lambda_{l l} m_{l}$.

The terms $k=l+1$ till $k=E-V+1$ remain unchanged, since they all correspond to edges that lie between vertices $v_{a}$ such that $v_{l}$ is before both $v_{a}$, and these $\tilde{\phi}_{a}$ are hence not shifted.

The terms $k=E-V+2$ till $k=E$ in (4.12), on the other hand, correspond to edges that lie between two vertices such that $v_{l}$ could be before the one and after the other. This is due to the fact that these edges do not belong to the maximal tree $\tau$ any longer. So in these terms, a shift by $\pm 2 \pi \lambda_{l l} m_{l}$ could have occurred by the shift of the integration range. But in all these terms, there is still a term $-2 \pi m_{k}$ present, and the sum over these $m_{k}$ is still performed. So, by appropriate shift of these summations, similar to those performed in the induction start, one can subsequently produce or erase terms of the form $\pm 2 \pi \lambda_{l l} m_{l}$ in all of the terms corresponding to $k=E-V+2$ till $k=E$. Since there are enough summations left, one has enough freedom to produce a $\pm 2 \pi \lambda_{l l} m_{l}$, where $\tilde{\phi}_{l}$ is present, or erase all terms with $m_{l}$, where $\tilde{\phi}_{l}$ is not present.

Thus, in the end, we again have a function only depending on $\tilde{\phi}_{l}-2 \pi \lambda_{l l} m_{l}$, and thus we can again apply formula (4.10), and, regardless of the sign of $\lambda_{l l}$, erase the infinite sum over $m_{l}$, obtaining an integration range of $\tilde{\phi}_{l}$ over all of $\mathbb{R}$, which results in (4.11). This proves the assertion of the lemma.

An immediate corollary of lemma 4.2 is that

$$
\begin{aligned}
\sqrt{\frac{2 \pi}{t}}^{E} \int_{[0,2 \pi]^{V}} & \frac{\mathrm{d} \tilde{\phi}_{1}}{2 \pi} \cdot \ldots \frac{\mathrm{d} \tilde{\phi}_{V}}{2 \pi} \sum_{m_{1}, \ldots, m_{E} \in \mathbb{Z}} \exp \left(-\sum_{k=1}^{E} \frac{\left(A_{k}+\lambda_{k a} \tilde{\phi}_{a}-2 \pi m_{k}\right)^{2}}{2 t}\right) \\
= & \sqrt{\frac{2 \pi}{t}} \int_{\mathbb{R}^{V-1}} \frac{\mathrm{d} \tilde{\phi}_{1}}{2 \pi} \cdot \ldots \cdot \frac{\mathrm{d} \tilde{\phi}_{V-1}}{2 \pi} \int_{0}^{2 \pi} \frac{\mathrm{d} \tilde{\phi}_{V}}{2 \pi} \\
& \times \sum_{m_{V}, \ldots, m_{E} \in \mathbb{Z}} \exp \left(-\sum_{k=1}^{V-1} \frac{\left(A_{k}+\lambda_{k a} \tilde{\phi}_{a}\right)^{2}}{2 t}-\sum_{k=V}^{E} \frac{\left(A_{k}+\lambda_{k a} \tilde{\phi}_{a}-2 \pi m_{k}\right)^{2}}{2 t}\right) .
\end{aligned}
$$

Note that one cannot perform the induction step with the integration over $\tilde{\phi}_{V}$ as well. The reason for this is that for the induction step it is crucial that it does not make sense to define whether $v_{V}$ is before or after any other vertex, since $e_{V}$ does not belong to the maximal tree $\tau$, in fact it does not even need to touch $v_{V}$. In particular, the integrand in (4.13) does not depend on $\tilde{\phi}_{V}$ at all! To see this, one only needs to shift all integrations $\tilde{\phi}_{1}, \ldots, \tilde{\phi}_{V-1}$ by $+\tilde{\phi}_{V}$. In all terms, the integration variables appear in the combination $\tilde{\phi}_{a}-\tilde{\phi}_{b}$ for any two different $a, b=1, \ldots, V$. So either we have the case that neither $a$ nor $b$ are equal to $V$, then nothing 
changes by this shift of integration, or one of $a$ or $b$ is equal to $V$. In this case the shift of the other one cancels the $\tilde{\phi}_{V}$, since both $\tilde{\phi}_{a}$ and $\tilde{\phi}_{b}$ appear with opposite sign. So, after this shift, $\tilde{\phi}_{V}$ occurs nowhere in the formula any more. Thus, we can perform the integration over $\tilde{\phi}_{V}$ trivially and obtain

$$
\text { (4.9) }=\left.\sqrt{\frac{2 \pi}{t}}^{E} \int_{\mathbb{R}^{V-1}} \frac{\mathrm{d} \tilde{\phi}_{1}}{2 \pi} \cdot \ldots \cdot \frac{\mathrm{d} \tilde{\phi}_{V-1}}{2 \pi} \sum_{m_{V}, \ldots, m_{E} \in \mathbb{Z}} \exp \left(-\sum_{k=1}^{E} \frac{\left(\tilde{A}_{k}+\lambda_{k a} \tilde{\phi}_{a}\right)^{2}}{2 t}\right)\right|_{\tilde{\phi}_{V}=0},
$$

where

$$
\tilde{A}_{k}:=\left\{\begin{array}{ll}
A_{k} & 1 \leqslant k \leqslant V-1 \\
A_{k}-2 \pi m_{k} & V \leqslant k \leqslant E
\end{array} .\right.
$$

To proceed, note that, since in every term in (4.14) the $\tilde{\phi}_{a}$ appear as pairs with opposite sign, the integrand is invariant under a simultaneous shift of all variables: $\tilde{\phi}_{a} \rightarrow \tilde{\phi}_{a}+c$. We use this fact to rewrite (4.14), by using the following technical lemma:

Lemma 4.3. Let $f: \mathbb{R}^{n} \rightarrow \mathbb{C}$ be a function with the symmetry

$$
f\left(x_{1}+c, \ldots, x_{n}+c\right)=f\left(x_{1}, \ldots, x_{n}\right) \quad \text { for all } c \in \mathbb{R}
$$

such that $x_{1}, \ldots, x_{n-1} \rightarrow f\left(x_{1}, \ldots, x_{n-1}, 0\right)$ is integrable. Then

$$
\int_{\mathbb{R}^{n-1}} \mathrm{~d} x_{1} \cdots \mathrm{d} x_{n-1} f\left(x_{1}, x_{2}, \ldots, x_{n-1}, 0\right)=n \int_{\mathbb{R}^{n}} \mathrm{~d} x_{1} \cdots \mathrm{d} x_{n} \delta\left(x_{1}+\cdots+x_{n}\right) f\left(x_{1}, \ldots, x_{n}\right) .
$$

Proof. The proof is elementary. Write

$$
\begin{aligned}
& \int_{\mathbb{R}^{n-1}} \mathrm{~d} x_{1}, \ldots \mathrm{d} x_{n-1} f\left(x_{1}, \ldots, x_{n-1}, 0\right) \\
& =\int_{\mathbb{R}^{n-1}} \mathrm{~d} x_{1}, \ldots \mathrm{d} x_{n-1} f\left(x_{1}-\frac{\sum_{k=1}^{n-1} x_{k}}{n}, \ldots, x_{n-1}-\frac{\sum_{k=1}^{n-1} x_{k}}{n},-\frac{\sum_{k=1}^{n-1} x_{k}}{n}\right) \\
& =\int_{\mathbb{R}^{n}} \mathrm{~d} x_{1}, \ldots \mathrm{d} x_{n} f\left(x_{1}-\frac{\sum_{k=1}^{n-1} x_{k}}{n}, \ldots, x_{n-1}-\frac{\sum_{k=1}^{n-1} x_{k}}{n}, x_{n}\right) \delta\left(x_{n}+\frac{\sum_{k=1}^{n-1} x_{k}}{n}\right) .
\end{aligned}
$$

Now perform a coordinate transformation

$$
\begin{aligned}
& \tilde{x}_{k}:=x_{k}-\frac{\sum_{k=1}^{n-1} x_{k}}{n}, \quad \text { for } \quad k=1, \ldots, n-1 \\
& \tilde{x}_{n}:=x_{n} .
\end{aligned}
$$

We have

$$
\sum_{n=1}^{n-1} \tilde{x}_{k}=\frac{\sum_{k=1}^{n-1} x_{k}}{n}
$$

and get

$\int_{\mathbb{R}^{n-1}} \mathrm{~d} x_{1}, \ldots \mathrm{d} x_{n-1} f\left(x_{1}, \ldots, x_{n-1}, 0\right)=\frac{1}{J} \int_{\mathbb{R}^{n}} \mathrm{~d}^{n} \tilde{x} f\left(\tilde{x}_{1}, \ldots, \tilde{x}_{n-1}, \tilde{x}_{n}\right) \delta\left(\tilde{x}_{1}+\ldots+\tilde{x}_{n}\right)$. 
Here $J=\operatorname{det}\left(\partial \tilde{x}_{k} / \partial x_{l}\right)$ is the Jacobian matrix of the coordinate transform. It is given by

$$
J=\operatorname{det}\left[\mathbb{1}_{n}-\frac{1}{n}\left(\begin{array}{ccccc}
1 & 1 & \cdots & 1 & 0 \\
1 & 1 & \cdots & 1 & 0 \\
\vdots & \vdots & \ddots & \vdots & \vdots \\
1 & 1 & \cdots & 1 & 0 \\
0 & 0 & \cdots & 0 & 0
\end{array}\right)\right]
$$

the determinant of which can easily computed to be $J=\frac{1}{n}$. Thus, with (4.17), the statement is proven.

We continue our analysis of the gauge-invariant overlap by using lemma (4.3) to rewrite (4.14) to obtain

$$
\text { (4.9) }=V \sqrt{\frac{2 \pi}{t}}^{E} \int_{\mathbb{R}^{V}} \frac{\mathrm{d} \tilde{\phi}_{1} \ldots \mathrm{d} \tilde{\phi}_{V}}{(2 \pi)^{V-1}} \delta\left(\sum_{a=1}^{V} \tilde{\phi}_{a}\right)_{m_{V}, \ldots, m_{E} \in \mathbb{Z}} \exp \left(-\sum_{k=1}^{E} \frac{\left(\tilde{A}_{k}+\lambda_{k a} \tilde{\phi}_{a}\right)^{2}}{2 t}\right) \text {. }
$$

Now we split the integrations over the $\tilde{\phi}_{a}$ from the $\tilde{A}_{k}$, in order to perform the integration. Because we are integrating over $\mathbb{R}^{V}$ and the integrand is holomorphic, we can now shift the $\tilde{\phi}_{a}$ also by complex amounts. This is necessary, since the $\tilde{A}_{k}$ are generically complex. A generic shift of the $\tilde{\phi}_{a}$ by complex numbers $z_{a}$ looks like

$$
\begin{aligned}
& \text { (4.9) }=V \sqrt{\frac{2 \pi}{t}}^{E} \int_{\mathbb{R}^{V}} \frac{\mathrm{d} \tilde{\phi}_{1} \ldots \mathrm{d} \tilde{\phi}_{V}}{(2 \pi)^{V-1}} \delta\left(\sum_{a=1}^{V}\left(\tilde{\phi}_{a}+z_{a}\right)\right) \\
& \times \sum_{m_{V}, \ldots, m_{E} \in \mathbb{Z}} \exp \left(-\sum_{k=1}^{E} \frac{\left(\tilde{A}_{k}+\lambda_{k a} \tilde{\phi}_{a}+\lambda_{k a} z_{a}\right)^{2}}{2 t}\right) \\
& =V \sqrt{\frac{2 \pi}{t}}^{E} \int_{\mathbb{R}^{V}} \frac{\mathrm{d} \tilde{\phi}_{1} \ldots \mathrm{d} \tilde{\phi}_{V}}{(2 \pi)^{V-1}} \delta\left(\sum_{a=1}^{V}\left(\tilde{\phi}_{a}+z_{a}\right)\right) \\
& \times \sum_{m_{V}, \ldots, m_{E} \in \mathbb{Z}} \exp \left[-\sum_{k=1}^{E}\left(\frac{\left(\lambda_{k a} \tilde{\phi}_{a}\right)^{2}}{2 t}+\frac{\lambda_{k a} \tilde{\phi}_{a}\left(\lambda_{k a} z_{a}+\tilde{A}_{k}\right)}{t}+\frac{\left(\lambda_{k a} z_{a}+\tilde{A}_{k}\right)^{2}}{2 t}\right)\right] \\
& =V \sqrt{\frac{2 \pi}{t}}^{E} \int_{\mathbb{R}^{V}} \frac{\mathrm{d} \tilde{\phi}_{1} \ldots \mathrm{d} \tilde{\phi}_{V}}{(2 \pi)^{V-1}} \delta\left(u^{T} \tilde{\phi}+u^{T} z\right) \\
& \times \sum_{m_{V}, \ldots, m_{E} \in \mathbb{Z}} \exp \left(-\frac{\tilde{\phi}^{T} \lambda \lambda^{T} \tilde{\phi}}{2 t}-\frac{\tilde{\phi}^{T} \lambda\left(\lambda^{T} z+\tilde{A}\right)}{t}-\frac{\left(\lambda^{T} z+\tilde{A}\right)^{T}\left(\lambda^{T} z+\tilde{A}\right)}{2 t}\right) \text {. }
\end{aligned}
$$

In (4.18), we have expressed all variables in terms of vectors and matrices, since this will simplify the handling of the expressions a lot. The vectors $u, \tilde{\phi}, z$ have length $V$, the vector $\tilde{A}$ has length $E$ and $\lambda$ is the $V \times E$ incidence matrix. The vector $u$ is given by (4.5). The $T$ means transpose.

The following lemma will help us to simplify this formula:

Lemma 4.4. Let $\lambda$ be the $V \times E$ incidence matrix of a connected graph $\gamma$ with $E$ edges and $V$ vertices, and $u=(11 \cdots 1)^{T}$ the vector of length $V$ containing only ones. For any vector 
$\tilde{A} \in \mathbb{C}^{E}$ the set of equations

$$
\begin{aligned}
& \lambda\left(\lambda^{T} z+\tilde{A}\right)=0 \\
& u^{T} z=0
\end{aligned}
$$

has exactly one solution in $\mathbb{C}^{V}$.

Proof. Rewrite the first of these equations as

$$
\lambda \lambda^{T} z=-\lambda \tilde{A} .
$$

Because of (4.6), $-\lambda \tilde{A}$ lies in the orthogonal complement of $u:-\lambda \tilde{A} \in\{u\}^{\perp}$. Since the graph $\gamma$ is connected, by Kirchhoff's theorem (4.1) the Kirchhoff-matrix $\lambda \lambda^{T}$ is positive definite on $\{u\}^{\perp}$, hence invertible on this $(V-1)$-dimensional subspace of $\mathbb{C}^{V}$. Define the $V \times V$ matrix $\sigma$ to be the inverse of $\lambda \lambda^{T}$ on $\{u\}^{\perp}$, and zero on $u$ :

$$
\begin{aligned}
& \sigma\left(\lambda \lambda^{T}\right) v=\left(\lambda \lambda^{T}\right) \sigma v=v \quad \text { for all } \quad u^{T} v=0 \\
& \sigma u=0 .
\end{aligned}
$$

So, the set of solutions of $\lambda \lambda^{T} z=-\lambda \tilde{A}$ is given by

$$
z=-\sigma \lambda \tilde{A}+\alpha u \quad \alpha \in \mathbb{C} .
$$

By the definition of $\sigma$, this means that

$$
z=-\sigma \lambda \tilde{A}
$$

is the unique solution of both equations, which proves the lemma.

Lemma 4.5. With the conditions of lemma 4.4, let $z$ be the unique solution of $\lambda\left(\lambda^{T} z+\tilde{A}\right)=0$ and $u^{T} z=0$, i.e. $z=-\sigma \lambda \tilde{A}$. Then

$$
-\lambda^{T} \sigma \lambda+1_{E}=P_{\operatorname{ker} \lambda},
$$

where $1_{E}$ is the $E \times E$ unit-matrix and $P_{\mathrm{ker} \lambda}$ is the orthogonal projector onto the subspace $\operatorname{ker} \lambda \subset \mathbb{C}^{E}$. In particular

$$
\lambda^{T} z+\tilde{A}=P_{\mathrm{ker} \lambda} \tilde{A} .
$$

Proof. Since

$$
\operatorname{ker} \lambda \oplus \operatorname{img} \lambda^{T}=1_{E} .
$$

The statement (4.21) can be rephrased as follows:

$$
\lambda^{T} \sigma \lambda=P_{\operatorname{img} \lambda^{T}},
$$

which is the projector onto the image of $\lambda^{T}$. Let $v \in \operatorname{img} \lambda^{T}$, so $v=\lambda^{T} w$ for some $w \in \mathbb{C}^{V}$. Even more, since $\lambda^{T} u=0$, we even can choose $w$ to be orthogonal to $u$ : $w \in\{u\}^{\perp}$. Then

$$
\lambda^{T} \sigma \lambda v=\lambda^{T} \sigma\left(\lambda \lambda^{T}\right) w=\lambda^{T} w=v
$$

since by definition $\sigma$ is the inverse of $\lambda \lambda^{T}$ on $\{u\}^{\perp}$.

Let, on the other hand, $v \in\left\{\operatorname{img} \lambda^{T}\right\}^{\perp}=\operatorname{ker} \lambda$. Then

$$
\lambda^{T} \sigma \lambda v=0
$$

trivially. Thus, $\lambda^{T} \sigma \lambda$ leaves vectors in $\operatorname{img} \lambda^{T}$ invariant and annihilates vectors from the orthogonal complement of $\operatorname{img} \lambda^{T}$. Hence $\lambda^{T} \sigma \lambda=P_{\operatorname{img} \lambda^{T}}$, from which it follows that

$$
-\lambda^{T} \sigma \lambda+1_{E}=P_{\text {ker } \lambda} \text {. }
$$


This was the first claim, the second one

$$
\lambda^{T} z+\tilde{A}=P_{\text {ker } \lambda} \tilde{A}
$$

follows immediately.

Lemmas 4.4 and 4.5 enable us to rewrite (4.18) as

$$
\text { (4.9) }=V \sqrt{\frac{2 \pi}{t}}^{E} \int_{\mathbb{R}^{V}} \frac{\mathrm{d} \tilde{\phi}_{1} \ldots \mathrm{d} \tilde{\phi}_{V}}{(2 \pi)^{V-1}} \delta\left(u^{T} \tilde{\phi}\right) \times \sum_{m_{V}, \ldots, m_{E} \in \mathbb{Z}} \exp \left(-\frac{\tilde{\phi}^{T} \lambda \lambda^{T} \tilde{\phi}}{2 t}-\frac{\tilde{A}^{T} P_{\operatorname{ker} \lambda} \tilde{A}}{2 t}\right) \text {. }
$$

We can now finally evaluate the gauge integrals in (4.25) with the help of Kirchhoff's theorem. Since the delta-function in the integrand of (4.25) assures that we only integrate over the orthogonal complement of $u$, instead of $\mathbb{R}^{V}$, and Kirchhoff's theorem 4.1 assures that the Kirchhoff-matrix $\lambda \lambda^{T}$ is positive definite there, we can immediately evaluate the integral

$$
\begin{aligned}
\int_{\mathbb{R}^{V}} \frac{\mathrm{d} \tilde{\phi}_{1} \cdots \mathrm{d} \tilde{\phi}_{V}}{(2 \pi)^{V-1}} \delta\left(u^{T} \tilde{\phi}\right) \exp \left(-\frac{\tilde{\phi}^{T} \lambda \lambda^{T} \tilde{\phi}}{2 t}\right) & =\sqrt{\frac{t}{2 \pi}} \frac{1}{\sqrt{\prod_{a=2}^{V} \mu_{a}}} \\
& =\frac{1}{\sqrt{G V}} \sqrt{\frac{t}{2 \pi}},
\end{aligned}
$$

where $\mu_{2}, \ldots, \mu_{V}$ are the nonzero eigenvalues of the Kirchhoff-matrix, and $G$ is the number of different possible maximal trees in the graph $\gamma$. With this, the gauge-invariant coherent state can be written as

$$
\text { (4.9) }=\sqrt{\frac{V}{G}} \sqrt{\frac{2 \pi}{t}}^{E-V+1} \sum_{m_{V}, \ldots, m_{E} \in \mathbb{Z}} \exp \left(-\frac{(A-2 \pi m)^{T} P_{\mathrm{ker} \lambda}(A-2 \pi m)}{2 t}\right),
$$

where $A=z-\phi$ is the vector containing $A_{k}=z_{k}-\phi_{k}$ in its $k$ th component, and $m$ being the vector containing 0 in the first $V-1$ components and $m_{V}, \ldots, m_{E}$ in the last $E-V+1$ components.

As already stated, the kernel of $\lambda$ is $E-V+1$ dimensional. Let $l_{1}, \ldots, l_{E-V+1}$ be an orthonormal basis of $\operatorname{ker} \lambda \subset \mathbb{R}^{E}$. Define

$$
z_{v}^{g i}:=l_{v}^{T} z, \quad \phi_{v}^{g i}:=l_{v}^{T} \phi, \quad m_{v}^{g i}:=l_{v}^{T} m .
$$

With this and $P_{\text {ker } \lambda}=\sum_{\nu=1}^{E-V+1} l_{v} l_{v}^{T}$, we get our final formula

$$
\Psi_{[z]}^{t}(\phi)=\sqrt{\frac{V}{G}} \sqrt{\frac{2 \pi}{t}}^{E-V+1} \sum_{m_{V}, \ldots, m_{E} \in \mathbb{Z}} \exp \left(-\sum_{\nu=1}^{E-V+1} \frac{\left(z_{\nu}^{g i}-\phi_{\nu}^{g i}-2 \pi m_{\nu}^{g i}\right)^{2}}{2 t}\right) .
$$

The gauge-invariant coherent state only depends on the $z_{v}^{g i}$ and $\phi_{v}^{g i}$, which are gauge-invariant combinations of the $z_{k}$ and $\phi_{k}$. That the linear combinations (4.27) are gauge-invariant, is clear from the construction, but one can immediately see this from the following: perform a gauge-transformation, which shifts the $\phi_{k}$ by $\lambda_{k a} \tilde{\phi}_{a}$. So, in matrices, one has $\phi \rightarrow \phi+\lambda^{T} \tilde{\phi}$. Thus,

$$
\phi_{v}^{g i}=l_{v}^{T} \phi \longrightarrow l_{v}^{T}\left(\phi+\lambda^{T} \tilde{\phi}\right)=l_{v}^{T} \phi+l_{v}^{T} \lambda^{T} \tilde{\phi}=l_{v}^{T} \phi=\phi_{v}^{g i}
$$

where $l_{v} \in \operatorname{ker} \lambda$ has been used, from which it follows that $\lambda l_{v}=0$, so $l_{v}^{T} \lambda^{T}=0$. Thus, the linear combinations of $\phi$ in $\phi_{\nu}^{g i}$ are all gauge invariant. The same holds true, of course, for the $z_{v}^{g i}$ and $m_{v}^{g i}$. So, the coherent states depend only on gauge-invariant combinations of $\phi$, which was clear from the beginning, but can now be seen explicitly. Note that the basis 
$\left\{l_{\nu}\right\}_{\nu=1}^{N-V+1}$ is, of course, not unique, but can be replaced by any other basis $l_{v}^{\prime}=R_{\nu \mu} l_{\mu}$ with $R \in O(E-V+1)$.

Compare the formula for the gauge-invariant coherent state (4.28) with the formula for the gauge-variant coherent states on $E$ edges (4.8). Up to a factor of $(V / G)^{1 / 2}$, the similarity is striking. One could be led to the conclusion that gauge-invariant coherent states are nothing but gauge-variant coherent states, only depending on gauge-invariant quantities. The fact that the gauge-invariant configuration space is diffeomorphic to $U(1)^{E-V+1}$, supports this guess.

However, this is not true. The reason is that the summation variables $m_{V}, \ldots, m_{E}$ are placed in wrong linear combinations in the formula. In particular, a gauge-invariant state is not

$$
\begin{aligned}
\Psi_{[z]}^{t}(\phi) \neq \sqrt{\frac{V}{G}} & \sqrt{\frac{2 \pi}{t}} \sum_{m_{1}^{g i}, \ldots, m_{E-V+1}^{g i} \in \mathbb{Z}}^{E-V+1} \exp \left(-\sum_{\nu=1}^{E-V+1} \frac{\left(z_{\nu}^{g i}-\phi_{\nu}^{g i}-2 \pi m_{v}^{g i}\right)^{2}}{2 t}\right) \\
& =\sqrt{\frac{V}{G}} \psi_{z^{g i}}^{t}\left(\phi^{g i}\right) .
\end{aligned}
$$

Of course, from the form (4.28) one cannot deduce a priori that the $m_{v}^{g i}$ could not, probably, be reordered in a way, maybe by an intelligent choice of $l_{v}$ and/or suitable shifting of summations, such that a form like (4.29), possibly with different $t$ for different variables, could be possible. But already simple examples like the 3-bridge graph show that this cannot be done. It could be, if one is lucky (in particular, on the 2-bridge graph), but generically a gauge-invariant coherent state is no complexifier coherent state depending on gauge-invariant variables.

\subsection{Peakedness of gauge-invariant coherent states}

In this chapter, we will shortly investigate the peakedness properties of the gauge-invariant coherent states. In particular, we will show that they are peaked on gauge-invariant quantities. Let $\gamma$ be a graph with $E$ edges. Then, a complexifier coherent state is then labeled by $E$ complex numbers $z_{1}, \ldots, z_{E}$ and a semiclassicality parameter $t>0$. Such a state is given by (4.8). The corresponding gauge-invariant coherent states, obtained by applying the projector onto the gauge-invariant sub-Hilbert space, has, in the last section, been shown to be (4.28).

Using $\left\langle\psi_{w}^{t} \mid \psi_{z}^{t}\right\rangle=\psi_{z-\bar{w}}^{2 t}(0)$, the inner product between two gauge-invariant coherent states $\Psi_{[w]}^{t}$ and $\Psi_{[z]}^{t}$ is given by

$\left\langle\Psi_{[w]}^{t} \mid \Psi_{[z]}^{t}\right\rangle=\sqrt{\frac{V}{G}} \sqrt{\frac{\pi}{t}}^{E-V+1} \sum_{m_{V}, \ldots, m_{E} \in \mathbb{Z}} \exp \left(-\sum_{\nu=1}^{E-V+1} \frac{\left(\bar{w}_{\nu}^{g i}-z_{\nu}^{g i}-2 \pi m_{\nu}^{g i}\right)^{2}}{4 t}\right)$.

With $z_{k}=\varphi_{k}-\mathrm{i} p_{k}$, i.e. by splitting the phase-space points into configuration and momentum variables, one immediately gets a formula for the norm of a gauge-invariant coherent state

$$
\left\|\Psi_{[z]}^{t}\right\|^{2}=\sqrt{\frac{V}{G}} \sqrt{\frac{\pi}{t}}^{E-V+1} \sum_{m_{V}, \ldots, m_{E} \in \mathbb{Z}} \exp \left(\sum_{\nu=1}^{E-V+1} \frac{\left(p_{v}^{g i}-\pi \mathrm{i} m_{v}^{g i}\right)^{2}}{t}\right) .
$$

Note that there is, apart from $m=0$, no combination of $m_{V}, \ldots, m_{E}$ such that the corresponding $m_{v}^{g i}=0$ for all $v=1, \ldots, E-V+1$. If there is one such combination, there are infinitely many of these combinations, hence infinitely many equally large terms. So, if there were, then the sum in (4.30) would not exist at all. But we know that the sum in (4.30) is absolutely convergent, so there is no such combination. 
What we just said is equivalent to saying that

$$
P_{\operatorname{ker} \lambda}\left(\begin{array}{c}
0 \\
\vdots \\
0 \\
m_{V} \\
\vdots \\
m_{E}
\end{array}\right) \neq 0 \quad \text { for all } m_{V}, \ldots m_{E} \in \mathbb{Z}
$$

which is, of course, due to the fact that the last $E-V+1$ components correspond, by construction, to the gauge-invariant directions on $U(1)^{E}$.

These considerations just show that, in the limit $t \rightarrow 0$, one can neglect all terms with $m_{v}^{g i} \neq 0$ in (4.31). In particular, we have

$$
\begin{aligned}
& \left|\left\|\Psi_{[z]}^{t}\right\|^{2}-\sqrt{\frac{V}{G}} \sqrt{\frac{\pi}{t}}^{E-V+1} \exp \left(\sum_{\nu=1}^{E-V+1} \frac{\left(p_{\nu}^{g i}\right)^{2}}{t}\right)\right| \\
& \leqslant \sqrt{\frac{V}{G}} \sqrt{\frac{\pi}{t}}^{E-V+1} \sum_{m_{V}, \ldots, m_{E} \in \mathbb{Z} \backslash\{0, \ldots, 0\}} \exp \left(\sum_{\nu=1}^{E-V+1} \frac{\left(p_{v}^{g i}\right)^{2}-\pi^{2}\left(m_{v}^{g i}\right)^{2}}{t}\right) \\
& =\sqrt{\frac{V}{G}} \sqrt{\frac{\pi}{t}}^{E-V+1} \exp \left(\sum_{\nu=1}^{E-V+1} \frac{\left(p_{\nu}^{g i}\right)^{2}}{t}\right)\left[\sum_{\vec{m} \in \mathbb{Z}^{E} \backslash\{0, \ldots, 0\}} \exp \left(-\sum_{\nu=1}^{E-V+1} \frac{\pi^{2}\left(m_{\nu}^{g i}\right)^{2}}{t}\right)\right] \\
& =\sqrt{\frac{V}{G}} \sqrt{\frac{\pi}{t}}^{E-V+1} \exp \left(\sum_{\nu=1}^{E-V+1} \frac{\left(p_{\nu}^{g i}\right)^{2}}{t}\right)\left[\sum_{\vec{m} \in \mathbb{Z}^{E} \backslash\{0, \ldots, 0\}} \exp \left(-\frac{\pi^{2} m^{T} P_{\mathrm{ker} \lambda} m}{t}\right)\right] \text {. }
\end{aligned}
$$

Define

$$
K:=\min _{\|m\|=1}\left\|P_{\mathrm{ker}} m\right\|>0 .
$$

With this, $m^{T} P_{\text {ker }} m \geqslant K^{2}\|m\|^{2}$, so we get

$$
\begin{aligned}
\sum_{m_{V}, \ldots, m_{E} \in \mathbb{Z}} \exp \left(-\pi^{2} \frac{m^{T} P_{\mathrm{ker} \lambda} m}{t}\right) & \leqslant \sum_{m_{V}, \ldots, m_{E} \in \mathbb{Z}} \exp \left(-\pi^{2} K^{2} \frac{\|m\|^{2}}{t}\right) \\
& =\left[\sum_{n \in \mathbb{Z}} \exp \left(\frac{-\pi^{2} K^{2}}{t} n^{2}\right)\right]^{E-V+1} \\
& =1+O\left(t^{\infty}\right),
\end{aligned}
$$

where the 1 comes from the $\vec{m}=\overrightarrow{0} \in \mathbb{Z}^{E}$ term. Thus, we can estimate (4.32) from the above by

$$
\begin{aligned}
\left|\left\|\Psi_{[z]}^{t}\right\|^{2}-\sqrt{\frac{V}{G}} \sqrt[\frac{\pi}{t}^{E-V+1}]{ } \exp \left(\sum_{\nu=1}^{E-V+1} \frac{\left(p_{v}^{g i}\right)^{2}}{t}\right)\right| \\
\leqslant \sqrt{\frac{V}{G} \sqrt{\frac{\pi}{t}}} \exp \left(\sum_{\nu=1}^{E-V+1} \frac{\left(p_{v}^{g i}\right)^{2}}{t}\right) O\left(t^{\infty}\right) .
\end{aligned}
$$


So the norm of a gauge-invariant coherent state is, as $t \rightarrow 0$, given by

$$
\left\|\Psi_{[z]}^{t}\right\|^{2}=\sqrt{\frac{V}{G}} \sqrt{\frac{\pi}{t}}^{E-V+1} \exp \left(\sum_{\nu=1}^{E-V+1} \frac{\left(p_{v}^{g i}\right)^{2}}{t}\right)\left(1+O\left(t^{\infty}\right)\right) .
$$

In a similar way, one can estimate the inner product of two gauge-invariant coherent states $\Psi_{[w]}^{t}$ and $\Psi_{[z]}^{t}$. It is given by (4.30), and if the labels $z_{v}^{g i}=\varphi_{\nu}^{g i}+i p_{v}^{g i}$ and $w_{v}^{g i}=\chi_{v}^{g i}+\mathrm{iq}_{\nu}^{g i}$ are nearly equal, then $\varphi_{v}^{g i}-\chi_{v}^{g i} \approx 0$, and again, only the $\vec{m}=\overrightarrow{0} \in \mathbb{Z}^{E}$-term dominates (4.30) in the limit $t \rightarrow 0$. We can then, along similar lines as in the computation of (4.31), estimate the inner product (4.30) in the limit $t \rightarrow 0$ to be

$$
\begin{aligned}
\left\langle\Psi_{[w]}^{t} \mid \Psi_{[z]}^{t}\right\rangle= & \sqrt{\frac{V}{G}} \sqrt{\frac{\pi}{t}}{ }^{E-V+1} \exp \left(-\sum_{\nu=1}^{E-V+1} \frac{\left(\varphi_{\nu}^{g i}-\chi_{\nu}^{g i}\right)^{2}}{4 t}\right. \\
& \left.+\mathrm{i} \sum_{\nu=1}^{E-V+1} \frac{\left(\varphi_{\nu}^{g i}-\chi_{\nu}^{g i}\right)\left(p_{\nu}^{g i}+q_{\nu}^{g i}\right)}{2 t}+\sum_{\nu=1}^{E-V+1} \frac{\left(p_{\nu}^{g i}+q_{\nu}^{g i}\right)^{2}}{4 t}\right)\left(1+O\left(t^{\infty}\right)\right) .
\end{aligned}
$$

For the overlap of two gauge-invariant coherent states $\Psi_{[w]}^{t}$ and $\Psi_{[z]}^{t}$ one has then, with (4.31)

$$
\begin{aligned}
I^{t}([w],[z])= & \frac{\left\langle\Psi_{[w]}^{t} \mid \Psi_{[z]}^{t}\right\rangle}{\left\|\Psi_{[w]}^{t}\right\|\left\|\Psi_{[z]}^{t}\right\|}=\exp \left(-\sum_{\nu=1}^{E-V+1} \frac{\left(\varphi_{\nu}^{g i}-\chi_{\nu}^{g i}\right)^{2}+\left(p_{\nu}^{g i}-q_{\nu}^{g i}\right)^{2}}{4 t}\right. \\
& \left.-\mathrm{i} \frac{\left(\varphi_{\nu}^{g i}-\chi_{\nu}^{g i}\right)\left(p_{v}^{g i}+q_{\nu}^{g i}\right)}{2 t}\right)\left(1+O\left(t^{\infty}\right)\right) .
\end{aligned}
$$

So the peakedness property can be read off immediately

$$
\left|I^{t}([w],[z])\right|^{2}=\exp \left(-\frac{\left\|P_{\operatorname{ker} \lambda}(z-w)\right\|^{2}}{2 t}\right)\left(1+O\left(t^{\infty}\right)\right) .
$$

This approaches 1 if the gauge-invariant quantities $[z] \approx[w]$ are close to each other, but as soon as $P_{\text {ker } \lambda}(z-w)$ goes away from zero, the expression becomes tiny, due to the tiny $t$. It follows that the overlap is peaked at coinciding gauge-invariant labels.

Due to the strong similarity of the gauge-invariant coherent states $\Psi_{[z]}^{t}$ to the complexifier coherent states $\psi_{z}^{t}$, it is quite easy to show that they satisfy the Ehrenfest property for gaugeinvariant observables. For this one can, up to terms of order $O\left(t^{\infty}\right)$, copy the corresponding proof for the complexifier coherent states [19]. However, the Ehrenfest property even follows generally from the peakedness property for any compact, semi-simple gauge group $G$, as will be shown in the following paper.

\section{Summary and conclusion}

This is the first of two papers concerning the gauge-invariant coherent states for loop quantum gravity. In this one, we have replaced the gauge group $G=S U(2)$ of LQG by the much simpler $G=U(1)$, the case $G=U(1)^{3}$, which is also of interest for LQG, follows immediately. We have investigated the gauge-invariant coherent states, in particular we have computed their explicit form and their overlap. The results found are very encouraging: while the complexifier coherent states are peaked on points in the kinematical phase space, which contains gauge information, the gauge-invariant coherent states, which are labeled by gauge-equivalence classes, are also sharply peaked on these. In particular, the overlap between two gaugeinvariant coherent states labeled with different gauge orbits tends to zero exponentially fast as 
the semiclassicality parameter $t$ tends to zero. Even more, it could be shown that the overlap is actually a Gaussian in the gauge-invariant variables.

This shows the good semiclassical properties of these states: as $t$ tends to zero, different states become approximately orthogonal very quickly, suppressing the quantum fluctuations between them. Also, the expectation values of operators corresponding to gauge-invariant kinematical observables (such as volume or area) are approximated well, which immediately follows from the corresponding properties of the gauge-variant CCS states.

This shows that the gauge-invariant coherent states are in fact useful for the semiclassical analysis of the gauge-invariant sector of LQG, and is the first step on the road to physical coherent states.

Apart from the nice semiclassical properties, the computation has revealed a deep connection of the gauge-invariant sector and the graph topology. In particular, the formula for the gauge-invariant coherent states on a graph $\gamma$ contains the incidence matrix $\lambda$ of $\gamma$. In contrast, the CCS are simply a product of states on each edge of the graph, hence have no notion of which edges are connected to each other and which are not, while the gauge-invariant coherent states explicitly contain information about the graph topology. This is simply due to the fact that the set of gauge-invariant degrees of freedom depend on the graph topology and can be computed by graph-theoretic methods.

While the results for $G=U(1)$ are quite encouraging, the case of ultimate interest for LQG is $G=S U(2)$, which is much more complicated. We will address this topic in the following paper, which will deal with this issue and try to establish as much results as possible from $U(1)$, where the problem could be solved completely and analytically, also for $S U(2)$.

\section{Acknowledgments}

BB would like to thank Hendryk Pfeiffer for the discussions about gauge-invariant functions and cohomology. Both authors would like to thank the referee for helpful comments. Research at the Perimeter Institute for Theoretical Physics is supported by the Government of Canada through NSERC and by the Province of Ontario.

\section{References}

[1] Ashtekar A, Lewandowski J, Marolf D, Mourao J and Thiemann T 1995 Quantization of diffeomorphism invariant theories of connections with local degrees of freedom J. Math. Phys. 366456 (arXiv: gr-qc/9504018)

[2] Thiemann T 2006 Introduction to Modern Canonical Quantum General Relativity (Cambridge: Cambridge University Press) (arXiv: gr-qc/0110034)

[3] Rovelli C 1998 Loop quantum gravity Living Rel. Rev. 11 (arXiv: gr-qc/9710008)

[4] Smolin L 2004 An invitation to loop quantum gravity, in Quantum Theory and Symmetries: Proc. 3rd Int. Symp. (Cincinnati, OH, 10-14 Sept 2003) ed P C Argyres, T J Hodges, F Mansouri, J J Scanio, P Suranyi and L C R Wijewardhana (Singapore: World Scientific) pp 655-82 (arXiv: hep-th/0408048)

[5] Lewandowski J, Okołow A, Sahlmann H and Thiemann T 2006 Uniqueness of diffeomorphism invariant states on holonomy-flux algebras Commun. Math. Phys. 267703 (arXiv: gr-qc/0504147)

[6] Henneuax M and Teitelboim C 1992 Quantization of Gauge Systems (Princeton, NJ: Princeton University Press)

[7] Baranger M, de Aguiar M A M, Keck F, Korsch H J and Schellhaaß B 2001 Semiclassical approximations in phase space with coherent states J. Phys. A: Math. Gen. 347227 (arXiv: quant-ph/0105153)

see also Baranger M, de Aguiar M A M, Keck F, Korsch H J and Schellhaaß B 2002 Semiclassical approximations in phase space with coherent states J. Phys. A: Math. Gen. 359493

Baranger M, de Aguiar M A M, Keck F, Korsch H J and Schellhaaß B 2003 Semiclassical approximations in phase space with coherent states J. Phys. A: Math. Gen. 369795 
[8] Mirbach B and Korsch H J 1995 Phase space entropy and global phase space structures of (chaotic) quantum systems Phys. Rev. Lett. 75362

[9] Wiescher H and Korsch H J 1997 Intrinsic ordering of quasienergy states for mixed regular/chaotic quantum systems: zeros of the Husimi distribution J. Phys. A: Math. Gen. 301763

[10] Klauder J R and Skagerstam B S 1985 Coherent States: Applications in Physics and Mathematical Physics (Singapore: World Scientific)

[11] Van Vleck J H 1928 The correspondence principle in the statistical interpretation of quantum mechanics Proc. Natl. Acad. Sci. USA 14178

[12] Glauber R J 1963 Coherent and incoherent states of the radiation field Phys. Rev. 1312766

[13] Hall B 1994 The Segal-Bargmann coherent state transform for compact Lie groups J. Funct. Anal. 122103 Hall B 1997 The inverse Segal-Bargmann transform for compact Lie groups J. Funct. Anal. 14398

[14] Hall B 1997 Phase space bounds for quantum mechanics on a compact Lie group Commun. Math. Phys. 184233

[15] Kastrup H 2006 Quantization of the canonically conjugate pair angle and orbital angular momentum Phys. Rev. A 73052104 (arXiv: quant-ph/0510234)

[16] Kowalski K, Rembieliński J and Papaloucas L C 1996 Coherent states for a quantum particle on a circle $J$. Phys. A: Math. Gen. 294149 (arXiv: quant-ph/9801029)

[17] Thiemann T 2001 Complexifier coherent states for quantum general relativity Class. Quantum Grav. 182025 (arXiv: gr-qc/0206037)

[18] Thiemann T 2001 Gauge field theory coherent states (GCS): I. General properties Class. Quantum Grav. 182025 (arXiv: hep-th/0005233)

[19] Thiemann T and Winkler O 2001 Gauge field theory coherent states (GCS): II. Peakedness properties Class. Quantum Grav. 182561 (arXiv: hep-th/0005237)

Thiemann T and Winkler O 2001 Gauge field theory coherent states (GCS): III. Ehrenfest theorems Class. Quantum Grav. 184629 (arXiv: hep-th/0005234)

[20] Giesel K and Thiemann T 2007 Algebraic quantum gravity (AQG): I. Conceptual setup Class. Quantum Grav. 242465 (arXiv: gr-qc/0607099)

Giesel K and Thiemann T 2007 Algebraic quantum gravity (AQG): II. Semiclassical analysis Class. Quantum Grav. 242499 (arXiv: gr-qc/0607100)

Giesel K and Thiemann T 2007 Algebraic quantum gravity (AQG): III. Semiclassical perturbation theory Class. Quantum Grav. 242565 (arXiv: gr-qc/0607101)

[21] Rovelli C and Smolin L 1995 Spin networks and quantum gravity Phys. Rev. D 525743 (arXiv: gr-qc/9505006)

[22] Varadarajan M 2005 The graviton vacuum as a distributional state in kinematic loop quantum gravity Class. Quantum Grav. 22 1207-38 (arXiv: gr-qc/0410120)

[23] Sahlmann H and Thiemann T 2006 Towards the QFT on curved spacetime limit of QGR: I. A general scheme Class. Quantum Grav. 23867 (arXiv: gr-qc/0207030)

Sahlmann H and Thiemann T 2006 Towards the QFT on curved spacetime limit of QGR: II. A concrete implementation Class. Quantum Grav. 23909 (arXiv: gr-qc/0207031)

[24] Biggs N 1993 Algebraic Graph Theory 2nd edn (Cambridge: Cambridge Mathematical Library) 